\title{
Quasi-bounded trees and analytic inductions
}

\author{
by
}

\author{
Jean Saint Raymond (Paris)
}

\begin{abstract}
A tree $T$ on $\omega$ is said to be cofinal if for every $\alpha \in \omega^{\omega}$ there is some branch $\beta$ of $T$ such that $\alpha \leq \beta$, and quasi-bounded otherwise. We prove that the set of quasi-bounded trees is a complete $\boldsymbol{\Sigma}_{1}^{1}$-inductive set. In particular, it is neither analytic nor co-analytic.
\end{abstract}

In a recent joint work with G. Debs, we were led to study the complexity of the set of cofinal trees as a subset of the compact set of all trees on $\omega$, in fact to show that this set is not $\boldsymbol{\Pi}_{1}^{1}$. The aim of this paper is to compute the exact complexity of this set, which appears to be beyond the $\sigma$-algebra generated by the analytic sets. We also prove similar results concerning the set of cofinal or quasi-bounded closed subsets of the Baire space with respect to the Effros Borel structure on the set $\mathcal{F}\left(\omega^{\omega}\right)$ of closed nonempty subsets of $\omega^{\omega}$.

Most of the definitions and results we recall here can be found in [4], which we refer to for all undefined notions and basic properties of classical descriptive classes.

Sequences and trees. For any set $E$ we denote by $\operatorname{Seq}(E)$ the set of finite sequences of elements of $E$. If $s=\left\langle e_{0}, e_{1}, \ldots, e_{k-1}\right\rangle \in \operatorname{Seq}(E)$ we denote by $|s|$ its length $k$. As usual, for any two $s=\left\langle e_{0}, e_{1}, \ldots, e_{k-1}\right\rangle$ and $t=\left\langle a_{0}, a_{1}, \ldots, a_{l-1}\right\rangle$ in $\operatorname{Seq}(E)$ we say that $t$ extends $s$ or that $s$ is a beginning of $t$, and write $s \prec t$ if $|s|<|t|$ and $e_{i}=a_{i}$ for $i<|s|$. And we write $s \preceq t$ iff $s \prec t$ or $s=t$. When $s \in \operatorname{Seq}(E)$ and $k \leq|s|$, we denote by $s_{\mid k}$ the sequence $s^{\prime}$ of length $k$ such that $s^{\prime} \preceq s$. Also we denote by $s \frown t$ the concatenation of $s$ and $t$, that is, the sequence $\left\langle e_{0}, e_{1}, \ldots, e_{k-1}, a_{0}, a_{1}, \ldots, a_{l-1}\right\rangle$ whose length is $|s|+|t|$.

For $s$ and $t$ in $\operatorname{Seq}(\omega)$ we write $s \leq t$ if $s$ and $t$ have the same length and moreover $s(i) \leq t(i)$ for every $i<|s|$.

2000 Mathematics Subject Classification: 03E15, 54H05.

Key words and phrases: dominating trees, induction, Borel games. 
We extend these notations to infinite sequences: for $\alpha=\left(a_{n}\right) \in E^{\omega}$ we denote by $\alpha_{\mid k}$ the sequence $t=\left\langle a_{0}, a_{1}, \ldots, a_{k-1}\right\rangle$, and write $t \prec \alpha$. For $s \in \operatorname{Seq}(E)$ of length $k$ and $\alpha \in E^{\omega}$ the concatenation $s \frown \alpha$ is the infinite sequence $\beta$ such that $s \prec \beta$ and $\beta(k+i)=\alpha(i)$ for all $i \in \omega$. It will also be convenient for $s \in \operatorname{Seq}(\omega), \alpha \in \omega^{\omega}$ and $\beta \in \omega^{\omega}$ to write $\alpha \leq \beta$ iff $\alpha(i) \leq \beta(i)$ for all $i$, and $s \leq \alpha$ iff $s \leq \alpha_{\mid k}$ where $k=|s|$.

For any countable set $I$ we identify the set $\mathcal{P}(I)$ of subsets of $I$ with the compact space $2^{I}=\{0,1\}^{I}$ by associating to each subset $J$ of $I$ its characteristic function $\chi_{J}: I \rightarrow\{0,1\}$. In particular, if $a$ and $b$ are two members of $2^{\omega}$, we will write $a \leq b$ as well as $a \subset b$.

By a tree $T$ on $E$ we mean a nonempty subset of $\operatorname{Seq}(E)$ which is left hereditary with respect to $\preceq$, that is, $(s \preceq t$ and $t \in T) \Rightarrow s \in T$. So the empty sequence $\emptyset$ belongs to any tree. An infinite branch (or a branch for short) of $T$ is an infinite sequence $\alpha \in E^{\omega}$ such that $\alpha_{\mid k} \in T$ for all $k$ (or equivalently for infinitely many $k$ 's). We denote by $\lceil T\rceil$ the set of branches of $T$, which is a closed subset of $E^{\omega}$ equipped with the product topology when $E$ itself has the discrete topology. Conversely, for any closed subset $F$ of $E^{\omega}$ there are trees $T$ such that $\lceil T\rceil=F$.

A tree $T$ is said to be well-founded if it has no infinite branch, and ill-founded otherwise.

A tree $T$ on $\omega$ is said to be monotone if whenever $s \leq t$ and $s \in T$ then $t \in T$. It is clear that if $T$ is monotone and $\alpha$ is any branch of $T$ then $\beta \in\lceil T\rceil$ whenever $\beta \in \omega^{\omega}$ and $\alpha \leq \beta$.

We denote by $\mathcal{T}$ the set of all trees on $\omega$ and by $\mathcal{T}^{+}$the set of all monotone trees on $\omega$, which are both closed subsets of $\mathcal{P}(\operatorname{Seq}(\omega))$, hence compact metrizable spaces. It is a well known and fundamental fact that the set WF of well-founded trees on $\omega$ is a complete $\boldsymbol{\Pi}_{1}^{1}$-subset of $\mathcal{T}$.

If $E$ and $F$ are two sets, a finite sequence $s$ of length $n$ of elements of $E \times F$ can be canonically identified with a pair $(t, u)$ with $t \in \operatorname{Seq}(E)$, $u \in \operatorname{Seq}(F)$ and $|t|=|u|=n$. Then a tree $T$ on $E \times F$ can be viewed as a set of pairs $(t, u)$ in $\operatorname{Seq}(E) \times \operatorname{Seq}(F)$ satisfying $|t|=|u|$. So we will say that $t \in \operatorname{Seq}(E)$ and $u \in \operatorname{Seq}(F)$ are $T$-compatible if $\left(t_{\mid k}, u_{\mid k}\right) \in T$, where $k=\min (|t|,|u|)$. In the same way, for $t \in \operatorname{Seq}(E)$ and $\beta \in F^{\omega}$, we say that $t$ and $\beta$ are $T$-compatible if $\left(t, \beta_{\mid k}\right) \in T$, where $k=|t|$.

It is easy to check that, for $\beta \in F^{\omega}$, the set

$$
T(\beta):=\{t \in \operatorname{Seq}(E): t \text { is } T \text {-compatible with } \beta\}
$$

is a tree on $E$ and that $\alpha \in\lceil T(\beta)\rceil$ if and only if $(\alpha, \beta) \in\lceil T\rceil$.

Inductions. Let $I$ and $P$ be sets, with $I$ countable. A mapping $\Phi$ : $\mathcal{P}(I) \times P \rightarrow \mathcal{P}(I)$ is called an induction if it is monotone with respect to the first variable for every $x \in P$, i.e., $a \subset b \subset I \Rightarrow \Phi(a, x) \subset \Phi(b, x)$. 
For such a mapping, one can define inductively on $\xi \in \omega_{1}$ subsets $\Phi^{\xi}(x)$ of $I$, for fixed $x \in P$, by

$$
\Phi^{0}(x)=\emptyset, \quad \Phi^{\xi+1}(x):=\Phi\left(\Phi^{\xi}(x), x\right) \quad \Phi^{\lambda}(x)=\bigcup_{\xi<\lambda} \Phi^{\xi}(x) \text { for limit } \lambda .
$$

It is easily shown that $\Phi^{\xi}(x) \subset \Phi^{\xi+1}(x)$ for all $\xi$, and $\Phi^{\eta}(x) \subset \Phi^{\xi}(x)$ for $\eta \leq \xi$. Since $I$ is countable, there is for each $x \in P$ a countable ordinal $\zeta$ such that $\Phi^{\zeta+1}(x)=\Phi^{\zeta}(x)$, thus $\Phi^{\xi}(x)=\Phi^{\zeta}(x)$ for all $\xi \geq \zeta$. We set $\Phi^{\infty}(x):=\Phi^{\zeta}(x)=\bigcup_{\xi \in \omega_{1}} \Phi^{\xi}(x)$. Thus $a:=\Phi^{\infty}(x)$ is a fixed point for $\Phi(\cdot, x)$, i.e. $\Phi(a, x)=a$. Conversely, if $a$ is any fixed point for $\Phi(\cdot, x)$, it is immediate by induction on $\xi$ that $\Phi^{\xi}(x) \subset a$ for all $x$, hence $\Phi^{\infty}(x) \subset a$. This implies that $\Phi^{\infty}(x)$ is the least fixed point for $\Phi(\cdot, x)$.

If $i^{*}$ is a fixed element of $I$, the inductive set $\operatorname{Ind}\left(\Phi, i^{*}\right)$ is defined as

$$
\operatorname{Ind}\left(\Phi, i^{*}\right):=\left\{x \in P: i^{*} \in \Phi^{\infty}(x)\right\}
$$

and it follows easily from what precedes that $x \notin \operatorname{Ind}\left(\Phi, i^{*}\right)$ is equivalent to

$$
\exists a \in \mathcal{P}(I) \quad i^{*} \notin a \text { and }(\forall i \in I \quad i \in a \text { or } i \notin \Phi(a, x)) .
$$

If $P$ is a Polish space and $\Gamma$ is a class, the induction $\Phi$ is said to be a $\Gamma$-induction if for every $i \in I$ the set $E_{i}:=\{(a, x): i \in \Phi(a, x)\}$ is a $\Gamma$-subset of $\mathcal{P}(I) \times P$, identified with the Polish space $2^{I} \times P$. In particular, if $\Phi$ is a $\boldsymbol{\Delta}_{1}^{1}$-induction, or even a $\boldsymbol{\Pi}_{1}^{1}$-induction, it follows immediately from (*) that $\operatorname{Ind}\left(\Phi, i^{*}\right)$ is $\boldsymbol{\Pi}_{1}^{1}$.

A subset $X$ of the Polish space $P$ is said to be $\boldsymbol{\Sigma}_{1}^{1}$-inductive if there is a countable set $I$, a $\boldsymbol{\Sigma}_{1}^{1}$-induction $\Phi$ on $\mathcal{P}(I) \times P$ and an $i^{*} \in I$ such that $X=\operatorname{Ind}\left(\Phi, i^{*}\right)$. We shall denote by $\boldsymbol{\Sigma}_{1}^{1}$-IND the class of $\boldsymbol{\Sigma}_{1}^{1}$-inductive sets.

The game quantifier. Let $P$ be a Polish space and $A$ a Borel subset of $\omega^{\omega} \times P$. For each fixed $x \in P$ the set $A_{x}:=\left\{\alpha \in \omega^{\omega}:(\alpha, x) \in A\right\}$ can be viewed as the payoff of a Borel game on $\omega$. So by Martin's Borel Determinacy Theorem this game $A_{x}$ is determined: if we denote by $\partial A$ the set

$$
\left\{x \in P \text { : Player I has a winning strategy in } A_{x}\right\},
$$

the complement of $\partial A$ in $P$ is the set

$$
\left\{x \in P \text { : Player II has a winning strategy in } A_{x}\right\},
$$

whence we deduce that both $\supset A$ and $P \backslash \supset A$ are $\boldsymbol{\Sigma}_{2}^{1}$.

If $\Gamma$ is a class of Borel sets, we denote by $\partial \Gamma$ the class $\{\partial A: A \subset$ $\left.\omega^{\omega} \times \omega^{\omega}, A \in \Gamma\right\}$. It is well known that $\partial \boldsymbol{\Sigma}_{1}^{0}=\boldsymbol{\Pi}_{1}^{1}$.

For $\Gamma=\boldsymbol{\Sigma}_{2}^{0}$, it follows from Wolfe's proof of $\boldsymbol{\Sigma}_{2}^{0}$ determinacy (see for example [4,6A.3]) that if $A \subset \omega^{\omega} \times P$ is $\boldsymbol{\Sigma}_{2}^{0}$ one can define an analytic induction $\Phi: \mathcal{P}(I) \times P \rightarrow \mathcal{P}(I)$ (where $I$ is the countable set $\{s \in \operatorname{Seq}(\omega)$ : $|s|$ even $\}$ ) such that Player I has a winning strategy in the game $A_{x}$ if and 
only if the empty sequence $\emptyset$ belongs to $\Phi^{\infty}(x)$. This shows that $\partial \boldsymbol{\Sigma}_{2}^{0} \subset$ $\boldsymbol{\Sigma}_{1}^{1}$-IND. Conversely, it was shown by R. Solovay (see [4, 7C.10]) that any $\boldsymbol{\Sigma}_{1}^{1}$-inductive set is $\partial \boldsymbol{\Sigma}_{2}^{0}$, that is, $\partial \boldsymbol{\Sigma}_{2}^{0}=\boldsymbol{\Sigma}_{1}^{1}$-IND.

Cofinal and quasi-bounded trees. As we said in the abstract, a tree $T$ on $\omega$ is said to be cofinal if for every $\alpha \in \omega^{\omega}$ there is an infinite branch $\beta$ of $T$ such that $\alpha \leq \beta$. We will say that such a branch $\beta$ is above $\alpha$.

If a tree $T$ is not cofinal there is an $\alpha \in \omega^{\omega}$ such that no branch of $T$ (if any) is above $\alpha$. Such an $\alpha$ need not be a bound for the branches of $T$, which would mean that "for all $\beta \in\lceil T\rceil, \beta \leq \alpha$ ", and we shall say that $\alpha$ is a quasi-bound for $T$, and that $T$ is quasi-bounded.

It is well known that trees on $\omega$ and closed subsets of $\omega^{\omega}$ are closely related. As above a subset $A$ of $\omega^{\omega}$ is said to be cofinal (sometimes also dominating) if for every $\alpha \in \omega^{\omega}$ there is some $\beta \geq \alpha$ in $A$. The subsets of $\omega^{\omega}$ which are not cofinal will also be called quasi-bounded. The structure of cofinal subsets of $\omega^{\omega}$ was already studied by several people (see [5], [1] or [2]).

The aim of this paper is to prove that the set QB of quasi-bounded trees on $\omega$ is a $\partial \boldsymbol{\Sigma}_{2}^{0}$-complete subset of $\mathcal{T}$. First we will prove that QB is $\partial \boldsymbol{\Sigma}_{2}^{0}$, hence $\boldsymbol{\Sigma}_{1}^{1}$-inductive. Then we will show that every $\boldsymbol{\Sigma}_{1}^{1}$-inductive subset of $\omega^{\omega}$ is continuously reducible to QB. This will complete the proof that QB is $\boldsymbol{\Sigma}_{1}^{1}$-IND-complete. In fact this will also prove that any $\boldsymbol{\Sigma}_{1}^{1}$-inductive set is $\partial \boldsymbol{\Sigma}_{2}^{0}$, hence will yield a new (but more complicated) proof of Solovay's result.

We will also consider the set QBC of closed quasi-bounded subsets of the Baire space, equipped with the Effros Borel structure. This set was already studied by S. Solecki ([5]), in connection with Haar null sets of a non-locally compact Polish group. He showed this set is $\boldsymbol{\Delta}_{2}^{1}$ but not $\boldsymbol{\Sigma}_{1}^{1}$. We shall prove here that it is $\boldsymbol{\Sigma}_{1}^{1}$-IND-complete.

There are only very few examples in the literature of true $\partial \boldsymbol{\Sigma}_{2}^{0}$ sets. The most important one is given by Kechris in [3], where he shows that $\boldsymbol{\Sigma}_{1}^{1}$-IND is the exact maximum complexity of $\sigma$-ideals of compact sets with $\boldsymbol{\Sigma}_{1}^{1}$ bases.

The main interest of our result is to yield a "natural" and combinatorially simple example of a $\partial \boldsymbol{\Pi}_{2}^{0}$ set. It could be used to prove that a set $X$ is not $\partial \Pi_{2}^{0}$ by reducing continuously QB to it, in the same way as one can prove that a set is not $\boldsymbol{\Sigma}_{1}^{1}$ by constructing a continuous reduction of WF to it.

Definition 1. For any tree $T$ on $\omega$, we denote by $T^{\circ}$ the tree defined by

$$
s \in T^{\circ} \Leftrightarrow\left(s=\emptyset \text { or }|s| \leq s(0) \text { or } s=\langle k\rangle \succ t \text { with } t_{\mid k} \notin T\right) .
$$

It is clear from the definition that if $\langle k\rangle \frown t$ belongs to $T^{\circ}$ and $k \leq l$ then $\langle l\rangle \frown t$ also belongs to $T^{\circ}$.

Lemma 2. Let $T$ be a monotone tree on $\omega$. Then the tree $T^{\circ}$ is quasibounded if and only if $T$ is ill-founded. Moreover, for any branch $\alpha$ of $T$, $\langle 0\rangle^{-} \alpha$ is a quasi-bound for $T^{\circ}$. 
Proof. Assume first $T$ is ill-founded and denote by $\alpha$ any branch of $T$. Then we claim that $\langle 0\rangle^{\frown} \alpha$ is a quasi-bound for $T^{\circ}$.

Indeed, assume by contradiction that $\langle k\rangle \frown \beta$ is a branch of $T^{\circ}$ above $\langle 0\rangle \frown \alpha$; then $t:=\beta_{\mid k} \notin T$. But since $\alpha \leq \beta$ we have $s:=\alpha_{\mid k} \leq \beta_{\mid k}=t$. So $s \in T$ since $\alpha \in\lceil T\rceil, t \notin T$ and $s \leq t$, in contradiction with $T \in \mathcal{T}^{+}$.

Assume now $T$ is well-founded and $\langle m\rangle \frown \alpha \in \omega^{\omega}$. We claim that $T^{\circ}$ possesses a branch above $\langle m\rangle \frown \alpha$.

Indeed, $\alpha \notin\lceil T\rceil=\emptyset$. Hence there is some integer $k$ such that $\alpha_{\mid k} \notin T$. Replacing $k$ by $\max (k, m)$ if necessary, we can assume $m \leq k$. Then $\langle k\rangle \frown \alpha$ is a branch of $T^{\circ}$, and $\langle m\rangle \frown \alpha \leq\langle k\rangle \frown \alpha$.

Theorem 3. The set $\mathrm{QB}$ is $\partial \boldsymbol{\Sigma}_{2}^{0}$.

Proof. Define the mapping $\psi: \operatorname{Seq}(2) \rightarrow \operatorname{Seq}(\omega)$ by counting the blocks of contiguous 0's inside $s$ : if $\psi(s)=\left\langle n_{0}, n_{1}, \ldots, n_{k-1}\right\rangle$ for some $s \in \operatorname{Seq}(2)$, then the sequence $s$ contains $k$ terms equal to 1 , with $n_{0}$ zeros before the first $1, n_{1}$ zeros between the first and the second $1, \ldots, n_{k-1}$ zeros between the last two 1's.

So $\psi$ is defined inductively by letting

$$
\left\{\begin{array}{l}
\psi(\emptyset)=\emptyset, \\
\psi(\langle 1\rangle)=\langle 0\rangle, \\
\psi(s \frown\langle 0\rangle)=\psi(s), \\
\psi(s \frown\langle 1,1\rangle)=\psi(s \frown\langle 1\rangle) \frown\langle 0\rangle, \\
\psi(s \frown\langle 1\rangle)=u \frown\langle p\rangle \Rightarrow \psi\left(s^{\frown}\langle 0,1\rangle\right)=u \frown\langle p+1\rangle .
\end{array}\right.
$$

Then it is clear that $|\psi(s)| \leq|s|$ and that for any two sequences $s$ and $s^{\prime}$ such that $s \prec s^{\prime}$ we have $\psi(s) \preceq \psi\left(s^{\prime}\right)$.

Denote by $P_{\infty}$ the set of those $\gamma^{\prime}$ s in $2^{\omega}$ which have infinitely many coordinates equal to 1 . For $\gamma \in P_{\infty}$ there is a unique $\beta \in \omega^{\omega}$ which we denote by $\widehat{\psi}(\gamma)$ such that $s \prec \gamma \Rightarrow \psi(s) \prec \beta$. It is easily checked and well known that $2^{\omega} \backslash P_{\infty}$ is countable and that $\widehat{\psi}$ is a homeomorphism from $P_{\infty}$ onto $\omega^{\omega}$.

For $T$ a given tree we define the game $G_{\mathrm{qb}}(T)$ where Player I plays integers $n_{0}, n_{1}, \ldots$, and Player II plays $c_{0}, c_{1}, \ldots$ in $\{0,1\}$ with the following two rules:

$R_{1}$ : for every $k, \psi\left(\left\langle c_{0}, c_{1}, \ldots, c_{k-1}\right\rangle\right) \in T$.

$R_{2}$ : for every $k,\left\langle n_{0}, n_{1}, \ldots, n_{p-1}\right\rangle \leq \psi\left(\left\langle c_{0}, c_{1}, \ldots, c_{k-1}\right\rangle\right)$, where $p$ is the length of $\psi\left(\left\langle c_{0}, c_{1}, \ldots, c_{k-1}\right\rangle\right)$.

The run where Player I plays $\left(n_{k}\right)$ and Player II plays $\left(c_{k}\right)$ is won by Player II iff $\left(c_{k}\right) \in P_{\infty}$. 
Clearly the set

$A:=\left\{\left(\left(n_{k}\right),\left(c_{k}\right), T\right):\right.$ Player II respects the rules and $\left.\left(c_{k}\right) \notin P_{\infty}\right\}$ is $\boldsymbol{\Sigma}_{2}^{0}$ in $\omega^{\omega} \times 2^{\omega} \times \mathcal{T}$. Hence the set $\partial A$ is $\partial \boldsymbol{\Sigma}_{2}^{0}$. Theorem 3 will then follow from the next two lemmas.

Lemma 4. If Player II has a winning strategy in the game $G_{\mathrm{qb}}(T)$, then the tree $T$ is continuously cofinal, i.e. there is a continuous function $f$ : $\omega^{\omega} \rightarrow\lceil T\rceil$ such that $f(\alpha) \geq \alpha$ for every $\alpha \in \omega^{\omega}$. In particular, $T$ is cofinal.

If $\tau$ is a winning strategy for Player II, it defines a continuous function $g: \omega^{\omega} \rightarrow 2^{\omega}$ such that for every $\alpha$ in $\omega^{\omega}$ and every $s=\left\langle n_{0}, n_{1}, \ldots, n_{k-1}\right\rangle \prec \alpha$ played by Player I the answer $\left\langle c_{0}, c_{1}, \ldots, c_{k-1}\right\rangle$ of Player II under $\tau$ satisfies $\left\langle c_{0}, c_{1}, \ldots, c_{k-1}\right\rangle \prec g(\alpha)$. It then follows from the rule $R_{1}$ that we have $\psi\left(\left\langle c_{0}, c_{1}, \ldots, c_{k-1}\right\rangle\right) \in T$. Moreover, since Player II wins, the run $g(\alpha)$ is in $P_{\infty}$. Hence $\widehat{\psi}(g(\alpha)) \in \omega^{\omega}$ and $\widehat{\psi}(g(\alpha))_{\mid p} \in T$ for arbitrarily large $p$, whence we conclude that $f(\alpha):=\widehat{\psi}(g(\alpha)) \in\lceil T\rceil$. Since $\psi$ is continuous on $P_{\infty}$, $f=\widehat{\psi} \circ g$ itself is continuous. Finally, it follows from the rule $R_{2}$ that $f(\alpha)_{\mid k} \geq \alpha_{\mid k}$ for arbitrarily large $k$, hence $f(\alpha) \geq \alpha$.

Lemma 5. If Player I has a winning strategy in $G_{\mathrm{qb}}(T)$, then $T$ is quasibounded.

If $\sigma$ is a winning strategy for Player I, it induces as above a continuous function $h: 2^{\omega} \rightarrow \omega^{\omega}$. Then the range $K:=h\left(2^{\omega}\right)$ is a compact subset of $\omega^{\omega}$, and one can define for all $n$ the integer $\alpha(n)=\sup _{x \in K} x(n)$. We claim that this $\alpha$ is a quasi-bound for $T$.

Indeed, if $\beta$ were a branch of $T$ such that $\alpha \leq \beta$, then Player II could play the following infinite run $\gamma$ : $\beta(0)$ times 0 , then 1 , then $\beta(1)$ times 0 , then $1, \ldots$ This would respect the rule $R_{1}$ since $\psi\left(\gamma_{\mid k}\right) \prec \beta$ for all $k$. And since $\gamma \in P_{\infty}$, we would have $\beta=\widehat{\psi}(\gamma)$. Moreover, since $h(\gamma) \in K$, we would have $h(\gamma) \leq \alpha \leq \beta=\widehat{\psi}(\gamma)$; this shows that the rule $R_{2}$ would also be respected. Finally, since $\gamma \in P_{\infty}$, Player II would win the run against the strategy $\sigma$. This contradiction completes the proof of the lemma.

Thus the proof of Theorem 3 is complete. One can notice that a similar game was used in [2] in order to prove that any cofinal $\boldsymbol{\Sigma}_{1}^{1}$ subset of $\omega^{\omega}$ is continuously cofinal.

REMARK. It follows from the previous proof that a quasi-bound for $T$ can be computed continuously from a winning strategy for Player I in $G_{\mathrm{qb}}(T)$. Conversely, a quasi-bound $\alpha$ for $T$ yields a simple strategy $\sigma$ for Player I: he plays $\alpha$ whatever Player II is answering. This strategy is clearly winning: in any run compatible with $\sigma$ a position $\left(\alpha_{\mid k},\left\langle c_{0}, c_{1}, \ldots, c_{k-1}\right\rangle\right)$ is 
reached for which no extension of $\psi\left(\left\langle c_{0}, c_{1}, \ldots, c_{k-1}\right\rangle\right)$ can be found in $T$ above $\alpha$; and beyond this position Player II must always play 0 .

We now intend to show that QB has complexity at least $\boldsymbol{\Sigma}_{1}^{1}$-IND.

TheOREM 6. If $X$ is a $\Sigma_{1}^{1}$-IND subset of $\omega^{\omega}$, there exists a continuous mapping $x \mapsto S(x)$ from $\omega^{\omega}$ to $\mathcal{T}$ such that $S(x) \in \mathrm{QB}$ if and only if $x \in X$.

Proof. Without loss of generality we assume that $\Phi: 2^{\omega} \times \omega^{\omega} \rightarrow 2^{\omega}$ is a $\boldsymbol{\Sigma}_{1}^{1}$-induction on $\omega$ and that

$$
x \in X \Leftrightarrow 0 \in \Phi^{\infty}(x) .
$$

Then for each $n$ the set $E_{n}:=\left\{(a, x) \in 2^{\omega} \times \omega^{\omega}: n \in \Phi(a, x)\right\}$ is $\boldsymbol{\Sigma}_{1}^{1}$ and there is some tree $T_{n}$ on $2 \times \omega \times \omega$ such that

$$
(a, x) \in E_{n} \Leftrightarrow \exists \beta \in \omega^{\omega} \quad(a, \beta, x) \in\left\lceil T_{n}\right\rceil
$$

where we identify the subset $\left\lceil T_{n}\right\rceil$ of $(2 \times \omega \times \omega)^{\omega}$ with a subset of $2^{\omega} \times \omega^{\omega} \times \omega^{\omega}$. Identifying $\operatorname{Seq}(2 \times \omega \times \omega)$ with the set

$$
\{(s, t, u) \in \operatorname{Seq}(2) \times \operatorname{Seq}(\omega) \times \operatorname{Seq}(\omega):|s|=|t|=|u|\}
$$

we now define trees $\widehat{T}_{n}$ and $U_{n}$ on $2 \times \omega \times \omega$ by

$$
\begin{aligned}
& (s, t, u) \in \widehat{T}_{n} \Leftrightarrow \exists s^{\prime} \exists t^{\prime} \quad s^{\prime} \leq s, t^{\prime} \leq t,\left(s^{\prime}, t^{\prime}, u\right) \in T_{n}, \\
& (s, t, u) \in U_{n} \Leftrightarrow\left\{\begin{array}{c}
(s, t, u)=(\emptyset, \emptyset, \emptyset) \\
\text { or }|s|=|t|=|u| \leq t(0) \\
\text { or else } t=\langle k\rangle-t^{*} \text { with }\left(s_{\mid k}, t_{\mid k}^{*}, u_{\mid k}\right) \notin \widehat{T}_{n} .
\end{array}\right.
\end{aligned}
$$

Fix a bijection $(n, p) \mapsto n * p$ from $\omega \times \omega$ onto $\omega$ which is separately increasing with respect to each variable and satisfies $n * 0 \leq 0 * n$. Then we necessarily have $0 * 0=0$. For example we can put

$$
n * p=\frac{(n+p)(n+p+1)}{2}+p .
$$

Then, for each $s \in \operatorname{Seq}(\omega)$ and each $n \in \omega$, we define the sequence $\theta_{n}(s) \in \operatorname{Seq}(\omega)$ by $\theta_{n}(s)=\langle s(n * 0), s(n * 1), \ldots s(n *(k-1))\rangle \quad$ where $n *(k-1)<|s| \leq n * k$. In particular we get $\theta_{n}(s)=\emptyset$ if $|s| \leq n * 0$. Define also $\theta^{*}(s) \in \operatorname{Seq}(2)$ by

$$
\theta^{*}(s)=\left\langle c_{0}, c_{1}, \ldots, c_{p-1}\right\rangle \text { where }\left\{\begin{array}{l}
(p-1) * 0<|s| \leq p * 0 \\
\text { and } c_{i}=1 \Leftrightarrow s(i * 0) \text { is odd }
\end{array}\right.
$$

Observe that for any $s \in \operatorname{Seq}(\omega)$ and any $n$, if $k=\left|\theta_{n}(s)\right|$ and $p=\left|\theta^{*}(s)\right|$, we have

$$
0 *(k-1) \leq n *(k-1)<|s| \leq p * 0 \leq 0 * p,
$$


hence $k-1<p$, thus $\left|\theta_{n}(s)\right| \leq\left|\theta^{*}(s)\right|$. Moreover it is clear that if $s \prec s^{\prime}$ we have $\theta_{n}(s) \preceq \theta_{n}\left(s^{\prime}\right)$ for all integers $n$, and $\theta^{*}(s) \preceq \theta^{*}\left(s^{\prime}\right)$. We extend $\theta_{n}$ and $\theta^{*}$ to $\omega^{\omega}$ by letting

$$
\begin{aligned}
& \widehat{\theta}_{n}(\alpha)(k)=\alpha(n * k), \\
& \widehat{\theta}^{*}(\alpha)(i)= \begin{cases}1 & \text { if } s(i * 0) \text { is odd }, \\
0 & \text { otherwise. }\end{cases}
\end{aligned}
$$

We now define, for $x \in \omega^{\omega}$, a tree $S(x)$ by

$$
s \in S(x) \Leftrightarrow\left\{\begin{array}{l}
s=\emptyset \text { or }\left(s(0)=0 \text { and } \forall n<\left|\theta^{*}(s)\right|\right. \\
\left.\left(\theta^{*}(s)(n)=1 \text { or }\left(\theta^{*}(s)_{\mid k}, \theta_{n}(s), x_{\mid k}\right) \in U_{n}\right)\right),
\end{array}\right.
$$

where $k=\left|\theta_{n}(s)\right|$.

The theorem will follow from the next four lemmas.

Lemma 7. The mapping $x \mapsto S(x)$ is continuous from $\omega^{\omega}$ to $\mathcal{T}$.

Proof. For any $s \in \operatorname{Seq}(\omega)$ define $k:=\left|\theta^{*}(s)\right|$. Then " $s \in S(x)$ " depends only on $x_{\mid k}$. Hence $\left\{x \in \omega^{\omega}: s \in S(x)\right\}$ is open and closed. This shows that the mapping $x \mapsto S(x)$ is continuous from $\omega^{\omega}$ to $\mathcal{T}$.

Lemma 8. For $a \in 2^{\omega}$ and $x \in \omega^{\omega}$ one has

$$
n \in \Phi(a, x) \Leftrightarrow \exists \beta \in \omega^{\omega} \quad(a, \beta, x) \in\left\lceil\widehat{T}_{n}\right\rceil \Leftrightarrow U_{n}(a, x) \in \mathrm{QB} .
$$

Moreover, if $\beta$ is any branch of $\widehat{T}_{n}(a, x)$, then $\langle 0\rangle \frown \beta$ is a quasi-bound for $U_{n}(a, x)$.

Proof. Notice that $T_{n} \subset \widehat{T}_{n}$. Thus if $n \in \Phi(a, x)$, then $(a, x) \in E_{n}$, hence there exists a $\beta$ such that $(a, \beta, x) \in\left\lceil T_{n}\right\rceil \subset\left\lceil\widehat{T}_{n}\right\rceil$.

Conversely, if $(a, \beta, x) \in\left\lceil\widehat{T}_{n}\right\rceil$ then for every integer $k,\left(a_{\mid k}, \beta_{\mid k}, x_{\mid k}\right)$ belongs to $\widehat{T}_{n}$. Hence there are $s \in 2^{k}$ and $t \in \omega^{k}$ such that $\left(s, t, x_{\mid k}\right) \in T_{n}$, $s \leq a_{\mid k}$ and $t \leq \beta_{\mid k}$. It follows that the set

$$
V:=\left\{(s, t, u) \in T_{n}:|s|=|t|=|u|, s \leq a, t \leq \beta, u \prec x\right\}
$$

is an infinite and finitely branching tree. By König's Lemma the tree $V$ is ill-founded. If $\left(a^{\prime}, \beta^{\prime}, x^{\prime}\right)$ is a branch of $V$, one necessarily has $a^{\prime} \leq a$ and $x^{\prime}=x$. Thus $\left(a^{\prime}, x\right) \in E_{n}$, hence $n \in \Phi\left(a^{\prime}, x\right) \subset \Phi(a, x)$.

Notice that for any $a \in 2^{\omega}$ and any $x \in \omega^{\omega}, U_{n}(a, x)=\widehat{T}_{n}(a, x)^{\circ}$ and $\widehat{T}_{n}(a, x)$ is monotone. Then it follows from Lemma 2 that $U_{n}(a, x)=$ $\widehat{T}_{n}(a, x)^{\circ}$ is quasi-bounded if and only if $\widehat{T}_{n}(a, x)$ is ill-founded, that is, if and only if $n \in \Phi(a, x)$.

Lemma 9. If $x \notin X$ then $S(x)$ is cofinal. 
Proof. Assume $x \notin X$ and let $a=\Phi^{\infty}(x)$. Then $0 \notin a$ and for all $n \notin a$ we have $n \notin \Phi(a, x)$. Let $\alpha \in \omega^{\omega}$ and define $\alpha_{n}=\widehat{\theta}_{n}(\alpha)$ for all $n$. We will produce a branch $\beta$ of $S(x)$ such that $\beta \geq \alpha$.

For $n \in a$ we define $\beta_{n}=\alpha_{n}$. For $n \notin a$, since $U_{n}(a, x)$ is cofinal, by Lemma 8 we can find $\beta_{n} \in\left\lceil U_{n}(a, x)\right\rceil$ such that $\alpha_{n} \leq \beta_{n}$. Replacing if necessary $\beta_{n}(0)$ by $\beta_{n}(0)+1$, we can assume that $\beta_{n}(0)$ is odd for $n \in a$ and even for $n \notin a$. Then defining $\beta$ by

$$
\forall n \forall p \quad \beta(n * p)=\beta_{n}(p)
$$

we get $\widehat{\theta}_{n}(\beta)=\beta_{n} \geq \alpha_{n}=\widehat{\theta}_{n}(\alpha), \beta(0)=\beta_{0}(0)$ is even and $\widehat{\theta}^{*}(\beta)=a$.

It follows easily that $\beta \geq \alpha$ and that for each $l, \beta_{\mid l} \in S(x)$, hence $\beta \in\lceil S(x)\rceil$.

\section{Lemma 10. If $x \in X$ then $S(x)$ is quasi-bounded.}

Proof. If $x \in X$, then $0 \in \Phi^{\infty}(x)$, and we can define for every $n \in \Phi^{\infty}(x)$ the rank $\varrho_{n}:=\min \left\{\xi: n \in \Phi^{(\xi)}(x)\right\} \in \omega_{1}$ and then $a_{n}:=\left\{p \in \omega: \varrho_{p}<\varrho_{n}\right\}$. Thus, for $n \in \Phi^{\infty}(x)$, we have $n \in \Phi\left(a_{n}, x\right)$. It follows that $\widehat{T}_{n}\left(a_{n}, x\right)$ is illfounded. Then we can choose a branch $\alpha_{n}^{*}$ of $\widehat{T}_{n}\left(a_{n}, x\right)$ and let $\alpha_{n}:=\langle 0\rangle \frown \alpha_{n}^{*}$.

For $n \notin \Phi^{\infty}(x)$ we choose $\alpha_{n}$ equal to the null sequence 0. Finally, defining $\alpha$ by

$$
\forall n \forall p \quad \alpha(n * p)=\alpha_{n}(p)
$$

we get $\widehat{\theta}_{n}(\alpha)=\alpha_{n}$ for all $n$.

We claim that $\alpha$ is a quasi-bound for $S(x)$. Indeed, assuming by contradiction that $\beta$ is a branch of $S(x)$ above $\alpha$, we should have $\beta_{n}:=\widehat{\theta}_{n}(\beta) \geq$ $\widehat{\theta}_{n}(\alpha)=\alpha_{n}$. Then put $a:=\widehat{\theta}^{*}(\beta) \in 2^{\omega}$. Since $\beta \in\lceil S(x)\rceil$, we should have $\beta(0)$ even, hence $0 \in \Phi^{\infty}(x) \backslash a$. It follows that $\left\{\varrho_{n}: n \in \Phi^{\infty}(x) \backslash a\right\}$ should be nonempty. Thus there would be an integer $m \in \Phi^{\infty}(x) \backslash a$ such that $\varrho_{m}=\min \left\{\varrho_{n}: n \in \Phi^{\infty}(x) \backslash a\right\}$. In particular $m \notin a$, hence $\beta_{m} \in\left\lceil U_{m}(a, x)\right\rceil$.

By minimality of $\varrho_{m}$ we would have $a_{m}=\left\{p: \varrho_{p}<\varrho_{m}\right\} \subset a$, hence $m \in \Phi(a, x)$. Since $\alpha_{m}^{*} \in\left\lceil\widehat{T}_{m}\left(a_{m}, x\right)\right\rceil$, this would also imply that $\alpha_{m}^{*} \in$ $\left\lceil\widehat{T}_{m}(a, x)\right\rceil$, hence $\alpha_{m}$ would be a quasi-bound for $U_{m}(a, x)$ by Lemma 8 , in contradiction with $\alpha_{m} \leq \beta_{m}$ and $\beta_{m} \in\left\lceil U_{m}(a, x)\right\rceil$.

This completes the proof of Theorem 6 .

Quasi-bounded closed subsets of the Baire space. Now we are interested in closed subsets of $\omega^{\omega}$ and will denote by $\mathcal{F}\left(\omega^{\omega}\right)$ the set of nonempty closed subsets of $\omega^{\omega}$ which we equip with the Effros Borel structure. As for trees on $\omega$, we shall say that a closed subset $F$ of $\omega^{\omega}$ is cofinal if for every $\alpha \in \omega^{\omega}$ there is some $\beta \geq \alpha$ in $F$, and that $F$ is quasi-bounded otherwise. We shall say that $\alpha$ is a quasi-bound for $F$ if $F \cap\{\beta: \beta \geq \alpha\}=\emptyset$. 
We will denote by QBC the subset of $\mathcal{F}\left(\omega^{\omega}\right)$ consisting of the quasi-bounded closed subsets of $\omega^{\omega}$.

We shall show in the following theorem that QBC behaves with respect to Borel reducibility in the same way as QB does with respect to continuous reducibility.

TheOREM 11. QBC is $\boldsymbol{\Sigma}_{1}^{1}$-IND-complete.

This follows immediately from the next two lemmas.

Lemma 12. If $P$ is a Polish space and $F: P \rightarrow \mathcal{F}\left(\omega^{\omega}\right)$ a Borel mapping then $F^{-1}(\mathrm{QBC})$ is $\boldsymbol{\Sigma}_{1}^{1}$-inductive.

Proof. For each $s \in \operatorname{Seq}(\omega)$ we denote by $N_{s}$ the basic open set $\{\alpha \in$ $\left.\omega^{\omega}: s \prec \alpha\right\}$. For $x \in P$ define

$$
T(x):=\left\{s \in \operatorname{Seq}(\omega): N_{s} \cap F(x) \neq \emptyset\right\},
$$

which is clearly a tree on $\omega$ such that $[T(x)\rceil=F(x)$. By definition of the Effros Borel structure, $\left\{H: N_{s} \cap H \neq \emptyset\right\}$ is Borel in $\mathcal{F}\left(\omega^{\omega}\right)$, thus $\{x \in P$ : $s \in T(x)\}$ is Borel for all $s$. Hence the mapping $f: x \mapsto T(x)$ is Borel from $P$ to $\mathcal{T}$. It is immediate from the definitions that $f(x) \in \mathrm{QB} \Leftrightarrow F(x) \in \mathrm{QBC}$. So $F^{-1}(\mathrm{QBC})=f^{-1}(\mathrm{QB})$.

As QB is $\boldsymbol{\Sigma}_{1}^{1}$-inductive in $\mathcal{T}$, there is an analytic induction $\Phi: \mathcal{P}(\omega) \times \mathcal{T}$ $\rightarrow \mathcal{P}(\omega)$ such that $T \in \mathrm{QB} \Leftrightarrow 0 \in \Phi^{\infty}(T)$. For $a \in \mathcal{P}(\omega)$ and $x \in P$ define

$$
\Psi(a, x):=\Phi(a, f(x)) .
$$

Then $\Psi$ is an induction and clearly $\Psi^{\xi}(x)=\Phi^{\xi}(f(x))$ for each $\xi$, hence $\Psi^{\infty}(x)=\Phi^{\infty}(f(x))$ and

$$
\begin{aligned}
F(x) \in \mathrm{QBC} \Leftrightarrow f(x) \in \mathrm{QB} & \Leftrightarrow 0 \in \Phi^{\infty}(f(x)) \\
& \Leftrightarrow 0 \in \Psi^{\infty}(x) \Leftrightarrow x \in \operatorname{Ind}(\Psi, 0) .
\end{aligned}
$$

Then $n \in \Psi(a, x) \Leftrightarrow \exists T \in \mathcal{T}(T=f(x)$ and $n \in \Phi(a, T))$, whence we conclude that $\Psi$ is $\boldsymbol{\Sigma}_{1}^{1}$ and finally that $F^{-1}(\mathrm{QBC})$ is $\boldsymbol{\Sigma}_{1}^{1}$-inductive.

Lemma 13. If $X$ is a $\boldsymbol{\Sigma}_{1}^{1}$-IND subset of $\omega^{\omega}$, then there exists a Borel reduction of $X$ to $\mathrm{QBC}$.

Proof. By Theorem 6 there is a continuous function $S$ from $\omega^{\omega}$ to $\mathcal{T}$ such that $S(x) \in \mathrm{QB} \Leftrightarrow x \in X$. Denote for $n \in \omega$ by $z_{n}$ the null sequence of length $n$, and

$$
\widetilde{S}(x):=\left\{s \frown z_{n}: s \in S(x), n \in \omega\right\} .
$$

Clearly if $\alpha \in \omega^{\omega}$ is any sequence such that $\alpha(n)>0$ for all $n$, then for all $\beta \in \omega^{\omega}$ we have

$$
\beta \geq \alpha \text { and } \beta \in\lceil S(x)\rceil \Leftrightarrow \beta \geq \alpha \text { and } \beta \in\lceil\widetilde{S}(x)\rceil,
$$


hence $S(x) \in \mathrm{QB} \Leftrightarrow \widetilde{S}(x) \in \mathrm{QB}$. Since

$$
s \in \widetilde{S}(x) \Leftrightarrow\left(\exists k, l \leq|s| \quad s_{\mid k} \in S(x) \text { and } s=s_{\mid k} \frown z_{l}\right),
$$

one sees that $\widetilde{S}$ is continuous and that $X=\widetilde{S}^{-1}(\mathrm{QB})$. Then define $F(x):=$ $\lceil\widetilde{S}(x)\rceil$. It is immediate that $F(x)$ is a quasi-bounded closed subset of $\omega^{\omega}$ iff $\widetilde{S}(x) \in \mathrm{QB}$, i.e. iff $x \in X$. Finally, it is enough to notice that for each $s \in \operatorname{Seq}(\omega)$ and each $x \in \omega^{\omega}$,

$$
\begin{aligned}
& F(x) \cap N_{s} \neq \emptyset \Rightarrow s \in \widetilde{S}(x) \Rightarrow \forall n s \frown z_{n} \in \widetilde{S}(x) \\
& \Rightarrow s \frown \mathbf{0} \in\lceil\widetilde{S}(x)\rceil \Rightarrow F(x) \cap N_{s} \neq \emptyset,
\end{aligned}
$$

so that $\left\{x: N_{s} \cap F(x) \neq \emptyset\right\}$ is clopen, and hence $F$ is Borel.

\section{References}

[1] J. Brendle, G. Hjorth and O. Spinas, Regularity properties for dominating projective sets, Ann. Pure Appl. Logic 72 (1995), 291-307.

[2] G. Debs and J. Saint Raymond, Cofinal $\boldsymbol{\Sigma}_{1}^{1}$ and $\boldsymbol{\Pi}_{1}^{1}$ subsets of $\omega^{\omega}$, Fund. Math. 159 (1999), 161-193.

[3] A. Kechris, The descriptive set theory of $\sigma$-ideals of compact sets, in: Logic Colloquium '88 (Padova, 1988), Stud. Logic Found. Math. 127, North-Holland, Amsterdam, 1989, 117-138.

[4] Y. N. Moschovakis, Descriptive Set Theory, North-Holland, Amsterdam, 1980.

[5] S. Solecki, Haar null and non-dominating sets, Fund. Math. 170 (2001), 197-217.

[6] O. Spinas, Dominating projective sets in the Baire space, Ann. Pure Appl. Logic 68 (1994), 327-342.

Analyse Fonctionnelle

Institut de Mathématique de Jussieu

Boîte 186

4, place Jussieu

75252 Paris Cedex 05, France

E-mail: jsr@ccr.jussieu.fr

Received 15 November 2005;

in revised form 3 February 2006 